\title{
RESISTENCIA A Phytophthora capsici LEO. EN LÍNEAS DE CHILE HUACLE (Capsicum annuum L.)
}

\author{
RESISTANCE TO Phytophthora capsici LEO. \\ IN HUACLE PEPPER (Capsicum annuum L.) LINES
}

\section{Eduardo Palma-Martínez'1, V. Heber Aguilar-Rincón ${ }^{1 *}$, Tarsicio Corona-Torres' y Olga Gómez-Rodríguez ${ }^{2}$}

\begin{abstract}
'Postgrado de Recursos Genéticos y Productividad-Genética, Campus Montecillo, Colegio de Postgraduados. km 36.5 Carr. México-Texcoco. 56230, Moentecillo, Texoco, México. Tel. (595) 952-02-00 Ext. 1510. ²Postgrado de Fitosanidad, Colegio de Postgraduados-Campus Montecillo. km 36.5 Carr. México-Texcoco. 56230, Montecillo, Texoco, México. Tel. (595) 952-02-00 Ext. 1610.
\end{abstract}

*Autor para correspondencia (victorheber@gmail.com)

\section{RESUMEN}

Entre las enfermedades importantes del cultivo de chile (Capsicum annuum L. ) se encuentra la "marchitez" (Phytophthora capsici L.). Como en otras enfermedades, la resistencia genética es una estrategia importante para su control. Una de las fuentes donde se pueden encontrar genes de resistencia son los materiales criollos. En evaluaciones previas se han encontrado colectas de chile Huacle con resistencia a dicho patógeno. En el presente estudio se determinó la herencia de la resistencia a $P$. capsici de las líneas de chile tipo Huacle $33.3,34.1$ y 35.3. Se generaron poblaciones $F_{2}$ de las cruzas de las tres líneas con la variedad susceptible NuMex Joe E. Parker. La determinación de similitud entre los genes de resistencia de las líneas 33.3 y 35.3 se hizo mediante las cruzas directa y recíproca entre ellas y se generaron poblaciones $\mathrm{F}_{2}$. Las poblaciones $\mathrm{F}_{2}$ se inocularon con aislados de $P$. capsici, que en evaluaciones previas se comportaron como avirulentas a las tres líneas progenitoras tipo Huacle y virulentas a la variedad susceptible. Las plántulas segregantes en $\mathrm{F}_{2}$ fueron clasificadas en resistentes y susceptibles de acuerdo con una escala de severidad de 1 a 7 , y se analizaron con una prueba de Ji-cuadrada para determinar el número de genes de resistencia presentes en cada línea. Las líneas 33.3 y 35.3 mostraron una segregación 3:1 de plantas resistentes y susceptibles, lo que indica la presencia de un gen distinto en cada una de ellas, resultado que se confirmó con la segregación 15:1 obtenida en las poblaciones $F_{2}$ de las cruzas entre estas líneas. La línea 34.1 presentó dos genes de resistencia dominantes, debido a la segregación 15:1 en la población $F_{2}$ entre plantas resistentes y susceptibles.

Palabras clave: Capsicum annuum, Phytophthora capsici, marchitez del chile, materiales criollos, resistencia genética.

\section{SUMMARY}

Among the major diseases of chili pepper cultivation is the "wilting" (Phytophthora capsici L.). As in other diseases, genetic resistance is an important strategy for its control. One of the sources where resistance genes can be found are local landraces. Huacle pepper accessions with resistace to this pathogen have been found in previous collections. In the present study, the inheritace of resistance to $P$. capsici of Huacle pepper lines 33.3, 34.1 and 35.3 was determined. $F_{2}$ populations were generated from crosses of the three lines with the susceptible variety NuMex Joe E. Parker. The determination of similarity between the resistance genes of lines 33.3 and 35.3 was accomplished through direct and reciprocal crosses between them and $F_{2}$ populations were generated. $F_{2}$ populations were inoculated with $P$. capsici isolates which in previous evaluations behaved as avirulent to the three Huacle parental lines and virulent to the susceptible variety. Segregant seedlings in $F_{2}$ were classified as resistant or susceptible, according to a severity scale of 1 to 7, and analyzed with a Chi-square test to determine the number of resistant genes present in each line. Lines 33.3 and 35.3 showed a 3:1 segregation ratio of resistant and susceptible seedlings which indicates the presence of a different gen in each line; result that was confirmed by the 15:1 segregation ratio observed in the $F_{2}$ populations generated from the crosses among these lines. Line 34.1 presented two dominant genes of resistance, due to the 15:1 segregation in the $F_{2}$ population between resistant and susceptible plants.

Index words: Capsicum annuum, Phytophthora capsici, pepper wilting, landraces, genetic resistance.

\section{INTRODUCCIÓN}

El chile (Capsicum spp.) es un cultivo con gran demanda alrededor del mundo, sus frutos son consumidos como alimento en varios países y sus colorantes son aprovechados por la industria farmacéutica y alimenticia (Aguilar et al., 2010; Wang y Bosland, 2006). El género Capsicum consta de 30 especies, de las cuales se han domesticado C. annuum, $C$. chinense, $C$. frutescens, $C$. baccatum y $C$. pubescens (Moscone et al., 2007; Pickersgill, 1984). En México se encuentra la mayor diversidad de la especie $C$. annuum y para su cultivo se destinan 152 mil hectáreas, con una producción de 2.2 millones de toneladas (SIAP, 2016).

Entre los principales problemas que presenta el cultivo se encuentran las enfermedades causadas por hongos, oomicetos, bacterias y virus, que son una limitante en la producción de chile. De estos patógenos, aquellos que se encuentran en el suelo como hongos y oomicetos son los de mayor riesgo para el cultivo (Sanogo y Carpenter, 2006; Velásquez-Valle et al., 2001; Velásquez-Valle y AmadorRamírez, 2007). En 1922 Leonian describió a Phytophthora capsici como responsable de la marchitez del chile en Nuevo México, enfermedad policíclica, altamente destructiva que provoca pérdidas del 60 \% o más (Castro et al., 2012; Silva-Rojas et al., 2009). Generalmente el manejo de la marchitez se lleva a cabo mediante la aplicación de productos biológicos y químicos (Fernández-Pavía et al., 
2004; González et al., 2009; Guigón-López y GonzálezGonzález, 2004).

El control de las enfermedades a través de variedades resistentes es una alternativa para reducir los daños producidos por P. capsici, además de no repercutir en el ambiente (Castro et al., 2012; Lamour et al., 2012; Reifschneider et al., 1992). Para desarrollar estas variedades es necesario encontrar materiales con características de resistencia como son en muchos casos las poblaciones nativas. Por otro lado, es necesario conocer la herencia de la resistencia al patógeno con el objeto de aplicar una adecuada estrategia de mejoramiento genético (Bartual et al., 1993; Morán-Bañuelos et al., 2010; Walker y Bosland, 1999).

Existen accesiones de chile reportados con cierto nivel de resistencia a P. capsici, por ejemplo: "PI 188476", "PI 201232", "PI 201234", "Chile Ancho San Luis", "Fyuco" y "Línea 29" (Candole et al., 2010; Kimble y Grogan, 1960; Roig et al., 2009; Walker y Bosland, 1999). Algunos chiles del tipo serrano provenientes del estado de Morelos, México han presentado un nivel de resistencia elevado, como es el caso del Criollo de Morelos 334 (CM-334) que se ha utilizado en distintas investigaciones referentes a la genética de la resistencia a P. capsici (Gil et al., 1991; Walker y Bosland, 1999). En los últimos años se han determinado loci de rasgos cuantitativos (QTL) en regiones de los cromosomas 4, 5, 6, 11 y 12 involucradas en la resistencia de CM-334; debido a esto se ha considerado que la resistencia es de tipo cuantitativa (Ogundiwin et al., 2005; Thabuis et al., 2003).

En el Colegio de Postgraduados se han colectado y caracterizado morfológicamente alrededor de 600 accesiones de los principales tipos de chiles cultivados en los estados de mayor producción, así como algunos criollos regionales poco conocidos y utilizados solamente de manera local (Corona, 2016; Com. pers.) ${ }^{1}$. A partir de los estudios de caracterización morfológica se han obtenido colectas centrales de chile que han sido evaluadas, entre otras cosas, por su resistencia a P. capsici; de esta forma se han encontrado, entre otras, tres accesiones del criollo regional tipo Huacle de Oaxaca con resistencia, de las cuales se desconoce su herencia. Por consiguiente, el objetivo del presente trabajo fue determinar la herencia de la resistencia a P. capsici de las líneas de chile Huacle 33.3, 34.1 y 35.3.

\section{MATERIALES Y MÉTODOS}

\section{Material vegetal}

Se utilizaron las líneas de chile Huacle 33.3, 34.1 y 35.3, proporcionadas por los doctores Víctor Heber Aguilar Rincón y Tarsicio Corona Torres, las cuales se obtuvieron por medio de autofecundaciones de plantas de tres colectas que en un estudio previo mostraron resistencia a $P$. capsici (Gómez-Rodríguez et al., 2017). La variedad NuMex Joe E. Parker® (NumexJP), como en otras investigaciones (Villar-Luna et al., 2009), se empleó como progenitor susceptible.

\section{Aislamientos utilizados}

Las cepas de Phytophthora capsici utilizadas fueron: ZAC (Zacatecas), J10, JC11 (Michoacán), proporcionadas por la Dra. Sylvia Fernández Pavía de la Universidad Michoacana de San Nicolás de Hidalgo. Las tres cepas se comportaron como avirulentas en las líneas 33.3, 34.1 y 35.3 de chile Huacle (Gómez-Rodríguez et al., 2017).

\section{Obtención de poblaciones $F_{2}$}

Para la obtención de las poblaciones $F_{2^{\prime}}$ en condiciones de invernadero pasivo, en el ciclo agrícola 2013 se establecieron cinco plantas de cada una de las líneas 33.3, 34.1 y 35.3, así como de la variedad susceptible NuMex Joe E. Parker ${ }^{\circledR}$. Se realizaron cruzas de cada una de las líneas con la variedad susceptible, al igual que entre las líneas 33.1 y 35.3, en este último caso las cruzas fueron tanto directas como recíprocas. En el ciclo agrícola 2014 se establecieron bajo las mismas condiciones de invernadero 12 plantas $F_{1}$ de cada una de las cruzas anteriores. Al llegar a floración en las plantas $F_{1}$ se realizaron cinco autofecundaciones en cada una de ellas para obtener las poblaciones $F_{2}$.

\section{Incremento del inóculo}

Para el incremento de inóculo de cada aislamiento utilizado se siguió la metodología propuesta por FernándezPavía et al. (2004), con modificaciones: cada aislamiento se cultivó in vitro por $10 \mathrm{~d}$ en cajas Petri con medio agar-jugo V8ß a $27^{\circ} \mathrm{C}$; después del tiempo de incubación, a cada caja Petri se le agregaron $10 \mathrm{~mL}$ de solución isotónica de cloruro de sodio DELMED ${ }^{\circledR}$ al $0.9 \%$ por 10 min, la cual se eliminó e inmediatamente después se adicionó agua destilada estéril y se expusieron a luz blanca continua por $72 \mathrm{~h}$ para promover la formación de esporangios. Las cajas con esporangios recibieron un choque térmico de 4 y $27^{\circ} \mathrm{C}$ por 30 min en cada temperatura, para promover la liberación de zoosporas en el medio acuoso. El líquido de cada caja se recuperó en un recipiente de vidrio para cuantificar el número de zoosporas por $\mathrm{mL}$ con ayuda de una cámara de Neubauer (Marca Neubauer-imp®, Marienfeld, Alemania). La suspensión se ajustó a $10^{5}$ zoosporas $\mathrm{mL}^{-1}$.

'Dr. Tarsicio Corona Torres (2016), especialista en fitogenética, Programa Genética, Colegio de Postgraduados. 


\section{Evaluación de la resistencia de poblaciones $F_{2}$}

Se sembraron 20 semillas de las líneas 33.5, 35.3 y de variedad NumexJP y 100 semillas de cada una de las cinco poblaciones $F_{2}$ para cada uno de los aislamientos con la que fueron evaluadas. Las semillas se desinfectaron con una solución de hipoclorito de sodio (Cloralex®) al 1 \% por 2 min y se colocaron en cajas de plástico con papel húmedo en su interior, incubándose a $28 \pm 1{ }^{\circ} \mathrm{C}$ durante 15 d. A los $12 \mathrm{~d}$ de germinadas las semillas, las plántulas se trasplantaron a vasos de plástico de $25 \mathrm{~cm}^{3}$ de capacidad con sustrato a base de arena fina esterilizada con vapor de agua por $2 \mathrm{~h}$. Se aplicó riego cada 24 h y la fertilización se llevó a cabo con la solución nutritiva Multiproposito® (1818-18 + microelementos) dos veces por semana.

El crecimiento se realizó en condiciones de ambiente controlado a una temperatura de $26 \pm 1{ }^{\circ} \mathrm{C}$, con un fotoperiodo de $14 \mathrm{~h}$ luz con una intensidad luminosa de 6768 lux (luz fluorescente) y $10 \mathrm{~h}$ de obscuridad. A las cuatro semanas después del transplante, las inoculaciones se realizaron aplicando al cuello de cada planta $1 \mathrm{~mL}$ de solución acuosa con una concentración de $10^{5}$ zoosporas. Antes de la inoculación se aplicó un riego a saturación para promover un microambiente de alta humedad para favorecer la infección.

Se registró la severidad en los progenitores resistentes y la variedad susceptible así como en 81 plantas de cada una de las poblaciones $F_{2}$ a través de una escala de categorías nominales arbitrarias del aspecto general de la planta propuesta por Sanogo (2006), con las siguientes modificaciones: 1 = sana, 2 = con necrosis en la base del tallo sin circundar, $3=$ con necrosis circundante en la base del tallo, $4=$ con necrosis circundante en la base del tallo y menos de $50 \%$ de defoliación, 5 = con necrosis circundante en la base del tallo y más de $50 \%$ de defoliación, $6=$ marchita, 7 = muerta. Las evaluaciones iniciaron tres días después de la inoculación (ddi) y continuaron cada 48 h durante 30 d. La relación de plantas $F_{2}$ resistentes/ susceptible se obtuvo al considerar plantas resistentes a aquellas con calificaciones de 1 a 3 y plantas susceptibles con calificaciones de 4 a 7 , de acuerdo con la escala de severidad descrita.

\section{Análisis estadístico}

La segregación se analizó mediante una prueba de icuadrada $\left(X^{2}\right)$, de acuerdo con las frecuencias observadas y esperadas de plantas resistentes/susceptibles con $a=$ 0.05. El análisis se realizó con los datos obtenidos a los siete ddi, momento en el cual todas las plantas del progenitor susceptible NumexJP se encontraban en el máximo nivel de la escala de severidad; es decir, muertas.

\section{RESULTADOS Y DISCUSIÓN}

Todas las plantas de la variedad NumexJP inoculadas con las cepas ZAC, J10 y JC11 presentaron necrosis en la base del tallo, defoliación, marchitez y finalmente murieron siete t. En cuanto a los progenitores resistentes, las plantas de la línea 33.3 inoculadas con las cepas ZAC, J10 y JC11 se comportaron como resistentes con excepción de una planta inoculada con la cepa ZAC (Cuadro 1). La presencia de una planta susceptible en la línea 33.3 con el aislamiento ZAC puede indicar una falta de uniformidad en la línea al no existir homocigosis porque esta línea lleva un solo ciclo de autofecundación. Las plantas de la línea 35.3 mostraron resistencia al ser inoculadas con los aislamientos ZAC y J10. El comportamiento de estas dos líneas de chile Huacle coincide con lo reportado por Candole et al. (2010) en la accesión "PI 201231" de chile Huacle, la cual presentó un porcentaje de plantas resistentes similar a CM-334 después de haber sido inoculadas con seis cepas de P. capsici.

La población $\mathrm{F}_{2} 33.3$ × NumexJP registró una proporción significativa de plantas resistentes/susceptibles 3:1 con Ios aislamientos J10 y ZAC; esta segregación puede estar relacionada con la presencia de un gen dominante de resistencia, mientras que para el aislamiento JC1 1 la proporción fue 15:1 que se puede deber a dos genes dominantes de resistencia. En la población $F_{2} 34.1 \times$ NumexJP inoculada con el aislamiento ZAC se observó una segregación 15:1 para dos genes dominantes de resistencia.

En el caso de las poblaciones $F_{2} 33.3$ y $34.1 \times$ NumexJP con segregaciones 15:1 se puede deber a la interacción entre razas diferentes del patógeno. Esta segregación ha sido reportada por Monroy-Barbosa y Bosland (2008) en líneas recombinantes de $\mathrm{CM}-334$ inoculadas con dos razas de P. capsici, lo que indica la acción independiente de dos genes. En la población $F_{2} 35.3$ × NumexJP inoculada con las cepas J10 y ZAC se encontró una segregación 3:1, correspondientes a un gen dominante de resistencia para estos dos aislamientos.

Las semejanzas en las proporciones observadas en poblaciones $F_{2} 33.3 \times$ NumexJP y $35.3 \times$ NumexJP, inoculadas con los aislamientos J10 y ZAC, sugieren una similitud entre los genes de resistencia de ambas líneas; sin embargo, en los resultados de la cruza directa y recíproca de estas dos líneas se observó una proporción de plantas resistentes/susceptibles para dos genes dominantes de resistencia o 15:1, lo que indica que los genes de resistencia presentes en las líneas 33.3 y 35.3 son distintos. Otro tipo de segregación encontrada en las poblaciones $F_{2}$ de las cruzas directa y recíproca fue 63:1 para tres genes dominantes de resistencia, aunque ésta no pudo ser 
Cuadro 1. Resultados obtenidos en los progenitores NuMex Joe E. Parker, líneas de chile Huacle y poblaciones $F_{2}$ inoculadas con tres aislamientos de $P$. capsici.

\begin{tabular}{|c|c|c|c|c|}
\hline Progenitores y Cruzas & Cepa de P. capsici & Plantas $\mathrm{R}: \mathrm{S}^{+}$ & Segregación R : S & $x^{2}$ \\
\hline \multirow[t]{3}{*}{ NuMexJP } & $\mathrm{J} 10$ & $0: 4$ & ---- & --- \\
\hline & ZAC & $0: 4$ & ---- & ---- \\
\hline & $\mathrm{JC11}$ & $0: 4$ & ---- & ---- \\
\hline \multirow[t]{3}{*}{33.3} & ZAC & $5: 1$ & ---- & ---- \\
\hline & J10 & $6: 0$ & ---- & ---- \\
\hline & JC11 & $6: 0$ & ---- & ---- \\
\hline \multirow[t]{2}{*}{35.3} & ZAC & $6: 0$ & ---- & ---- \\
\hline & $\mathrm{J} 10$ & $6: 0$ & ---- & ---- \\
\hline $\mathrm{F}_{2} 33.3 \times$ NuMexJP & ZAC & $54: 27$ & $3: 1$ & 3.00 \\
\hline $\mathrm{F}_{2} 33.3 \times$ NuMexJP & $\mathrm{JC11}$ & $72: 9$ & $15: 1$ & 3.26 \\
\hline $\mathrm{F}_{2} 33.3 \times$ NuMexJP & J10 & $54: 27$ & $3: 1$ & 3.00 \\
\hline $\mathrm{F}_{2} 34.1 \times$ NuMexJP & ZAC & $74: 7$ & $15: 1$ & 0.79 \\
\hline $\mathrm{F}_{2} 35.3 \times$ NuMexJP & J10 & $65: 16$ & $3: 1$ & 1.18 \\
\hline $\mathrm{F}_{2} 35.3 \times$ NuMexJP & ZAC & $65: 16$ & $3: 1$ & 1.18 \\
\hline$F_{2} 33.3 \times 35.3$ & J10 & $80: 1$ & $\begin{array}{l}15: 1 \\
63: 1\end{array}$ & $\begin{array}{l}3.47 \\
0.06\end{array}$ \\
\hline$F_{2} 35.3 \times 33.3$ & ZAC & $80: 1$ & $\begin{array}{l}15: 1 \\
63: 1\end{array}$ & $\begin{array}{l}3.47 \\
0.06\end{array}$ \\
\hline
\end{tabular}

${ }^{\dagger} \mathrm{R}: \mathrm{S}=$ Relación plantas resistentes : plantas susceptibles.

comprobada en las poblaciones $\mathrm{F}_{2}$ de la cruza de líneas resistentes 33.3 y 35.3 con la variedad susceptible NumexJP (Cuadro 1).

El tipo de resistencia encontrado en las líneas de chile Huacle no es fácil de definir. Si bien se encontraron segregaciones en las tres líneas que parecerían genes dominantes simples, en el patosistema ha sido difícil precisar con claridad el tipo de resistencia, debido a que existen resultados similares a los encontrados en esta investigación, donde la acción de genes simples son los responsables de la resistencia, como en el caso de las accesiones Fyuco, P51 y CM-334, donde un solo gen proporciona resistencia completa a una o más cepas de P. capsici (Barksdale et al., 1984; Walker y Bosland, 1999). Por otra parte, Reifschneider et al. (1992) determinaron en la accesión CNPH 148 de chile serrano una segregación 13:3 para un gen dominante y un gen recesivo involucrados en la resistencia. La diferencia entre los resultados se debe principalmente a la complejidad de la resistencia y se requerirá realizar investigaciones que den una mayor precisión en las bases genéticas de la resistencia de los chiles Huacles.

\section{CONCLUSIONES}

La resistencia a Phytophthora capsici que muestran las líneas de chile tipo Huacle 33.3 y 35.3 están determinadas por un gen dominante de resistencia en cada una de ellas. Los dos genes de resistencia presentes en estas dos líneas son genes distintos. A diferencia de las dos líneas anteriores, en la línea 34.1 se encuentran dos genes que determinan la resistencia a P. capsici.

\section{BIBLIOGRAFÍA}

Aguilar R. V. H., T. Corona T., P. López L., L. Latournerie M., M. Ramírez M., H. Villalón M. y J. A. Aguilar C. (2010) Los Chiles de México y su Distribución. SINAREFI, Colegio de Postgraduados, INIFAP, ITConkal, UANL, UAN. Montecillo, Texcoco, Estado de México. 
$114 \mathrm{p}$

Barksdale T. H., G. C. Papavizas and S. A. Johnston (1984) Resistance to foliar blight and crown rot of pepper caused by Phytophthora capsici. Plant Disease 68:506-509.

Bartual R., A. Lacasa, J. I. Marsal y J. C. Tello (1993) Efectos epistáticos en la resistencia a Phytophthora capsici Leon en pimiento (Capsicum annuum). Boletín de Sanidad Vegetal Plagas 19:485-490.

Candole B. L., P. J. Conner and P. Ji (2010) Screening Capsicum annuum accessions for resistance to six isolates of Phytophthora capsici. HortScience 45:254-259.

Castro R. A., S. P. Fernández P. y P. Osuna Á. (2012) Mecanismos de defensa del chile en el patosistema Capsicum annuum-Phytophthora capsici. Revista Mexicana de Fitopatología 30:49-65.

Fernández-Pavía S. P., C. L. Biles, M. E. Waugh, K. Onsurez-Waugh, G. Rodríguez-Alvarado and C. M. Liddell (2004) Characterization of Southern New Mexico Phytophthora capsici Leonian isolates from pepper (Capsicum annuum L.). Revista Mexicana de Fitopatología 22:82-89.

Gil O. R., C. Palazóon E. and J. Cuartero Z. (1991) Genetics of resistance to Phytophthora capsici in the pepper line 'SCM-334'. Plant Breeding 107:50-55.

Gómez-Rodríguez 0., T. Corona-Torres and V. H. Aguilar-Rincón (2017) Differential response of pepper (Capsicum annuum L.) lines to Phytophthora capsici and root-knot nematodes. Crop Protection 92:148-152.

González C. M. M., E. Villordo P., J. L. Pons H., F. Delgadillo S., R. Paredes M., H. Godoy H., J. L. Anaya L., F. P. Gámez V., T. Medina C., R. Rodríguez G., E. Ruiz C., A. Ruiz L., R. Cárdenas B., J. R. Cárdenas A., I. Torres P., E. Rendón P., J. Martínez S., F. Mojarro D., O. M. Villaseñor E. y B. Z. Guerrero A. (2009) Guía para el Manejo de la Marchitez del Chile en Guanajuato. CEPROCH Guanajuato, CONACYT, INIFAP y Gobierno del Estado de Guanajuato. Prometeo Editores. Guadalajara, Jalisco. $34 \mathrm{p}$.

Guigón L. C. y P. A. González G. (2004) Selección de cepas nativas de Trichoderma spp. con actividad antagónica sobre Phytophthora capsici Leonian y promotoras de crecimiento en el cultivo de chile (Capsicum annuum L.). Revista Mexicana de Fitopatología 22:117-124.

Kimble K. A. and R. G. Grogan (1960) Resistance to Phytophthora root rot in pepper. The Plant Disease Reporter 44:872-873.

Lamour K. H., R. Stam, J. Jupe and E. Huitema (2012) The oomycete broadhost-range pathogen Phytophthora capsici. Molecular Plant Pathology 13:329-337.

Monroy-Barbosa A. and P. W. Bosland (2008) Genetic analysis of Phytophthora root rot race-specific resistance in chile pepper. Journal of the American Society for Horticultural Science 133:825-829.

Morán-Bañuelos S. H., V. H. Aguilar-Rincón, T. Corona-Torres y E. ZavaletaMejía (2010) Resistencia a Phytophthora capsici Leo. de chiles nativos del sur de Puebla, México. Revista Fitotecnia Mexicana 33:21-26

Moscone E. A., M. A. Scaldaferro, M. Grabiele, N. M. Cecchini, Y. Sánchez G., R. Jarret, J. R. Daviña, D. A. Ducasse, G. E. Barboza and F. Ehrendorfer (2007) The evolution of chili peppers (Capsicum-Solanaceae): a cytogenetic perspective. Acta Horticulturae 745:137-170.

Ogundiwin E. A., T. F. Berke, M. Massoudi, L. L. Black, G. Huestis, D. Choi, S. Lee and J. P. Prince (2005) Construction of 2 intraspecific linkage maps and identification of resistance QTLs for Phytophthora capsici root-rot and foliar-blight diseases of pepper (Capsicum annuum L.). Genome 48:698-711.

Pickersgill B. (1984) Migration of chili peppers, Capsicum spp., in the Americas. In: Pre-Columbian Plant Migration. Vol. 76. D. Stone (ed.). Harvard University Press. Cambridge, Massachusetts. pp:105-123

Reifschneider F. J. B., L. S. Boiteux, P. T. Della Vecchia, J. M. Poulos and N. Kuroda (1992) Inheritance of adult-plant resistance to Phytophthora capsici in pepper. Euphytica 62:45-49.

Roig J. M., P. Occhiuto, R. J. Piccolo y C. R. Galmarini (2009) Evaluación de resistencia a Phytophthora capsici Leo. en germoplasma argentino de pimiento para pimentón. Horticultura Argentina 28:5-9.

Sanogo S. (2006) Predispositional effect of soil water saturation on infection of chile pepper by Phytophthora capsici. HortScience 41:172-175.

Sanogo S. and J. Carpenter (2006) Incidence of Phytophthora blight and Verticillium wilt within chile pepper fields in New Mexico. Plant Disease 90:291-296.

SIAP, Sistema de Información Agroalimentaria y Pesquera (2016) Cierre de la producción agrícola por cultivo. Sistema de Información Agroalimentaria y Pesquera. SAGARPA. México. http://www.siap. gob.mx/cierre-de-la-produccion-agricola-por-cultivo/ (Marzo 2016).

Silva-Rojas H. V., S. P. Fernández-Pavía, C. Góngora-Canul, B. C. Macías-López y G. D. Ávila-Quezada (2009) Distribución espacio temporal de la marchitez del chile (Capsicum annuum L.) en Chihuahua e identificación del agente causal Phytophthora capsici Leo. Revista Mexicana de Fitopatología 27:134-147.

Thabuis A., A. Palloix, S. Pflieger, A. M. Daubèze, C. Caranta and V. Lefebvre (2003) Comparative mapping of Phytophthora resistance loci in pepper germplasm: evidence for conserved resistance loci across Solanaceae and for a large genetic diversity. Theoretical and Applied Genetics 106:1473-1485.

Velásquez-Valle R., M. M. Medina-Aguilar y J. J. Luna-Ruiz (2001) Sintomatología y géneros de patógenos asociados con las pudriciones de la raíz del chile (Capsicum annuum L.) en el Norte-Centro de México. Revista Mexicana de Fitopatología 19:175-181.

Velásquez-Valle R. y M. D. Amador-Ramírez (2007) Análisis sobre la investigación fitopatológica de chile seco (Capsicum annuum L.) realizada por el Instituto Nacional de Investigaciones Forestales, Agrícolas y Pecuarias en los estados de Aguascalientes y Zacatecas, México. Revista Mexicana de Fitopatología 25:8084.

Villar-Luna E., B. Reyes-Trejo, R. I. Rojas-Martínez, O. Gómez-Rodríguez, A. M. Hernández-Anguiano y E. Zavaleta-Mejía (2009) Respuesta hipersensitiva en el follaje de chile CM-334 resistente a Phytophthora capsici infectado con Nacobbus aberrans. Nematropica 39:143-155.

Walker S. J. and P. W. Bosland (1999) Inheritance of Phytophthora root rot and foliar blight resistance in pepper. Journal of the American Society for Horticultural Science 124:14-18.

Wang D. and P. W. Bosland (2006) The genes of Capsicum. HortScience 41:1169-1187. 